\title{
Orbital pseudotumor: A single precursor clinical manifestation of Wegener granulomatosis in a ten year old boy
}

\author{
Paraskevi Maggina $^{{ }^{*}}$, Varvara Askiti ${ }^{2}$, Mersini Maurikou1, Maria Mila², Dionisios Papadatos ${ }^{1}$, \\ Lela Stamogianou ${ }^{1}$, Constantinos J. Stefanidis ${ }^{2}$ \\ ${ }^{1}$ Department of Pediatrics, “P. \& A. Kyriakou” Children’s Hospital, Athens, Greece \\ ${ }^{2}$ Department of Nephrology, “P. \& A. Kyriakou” Children’s Hospital, Athens, Greece \\ Email: *vivian.maggina@hotmail.com
}

Received 8 September 2011; revised 6 January 2012; accepted 30 January 2012

\begin{abstract}
Wegener granulomatosis (WG) is a type of vasculitis characterized by the presence of anti-neutrophil cytoplasmic antibodies (ANCA) and inflammation of small and medium sized vessels with granulomas formation. Most commonly affected organs include upper and lower respiratory tract, kidneys, eyes, nervous system and skin. Kidneys' involvement has a central position in the classification, diagnosis, treatment and prognosis of patients with WG, and is characterized by the presence of necrotic glomerulonephritis and clinical manifestations that vary from microscopic hematuria to acute renal insufficiency. We describe a case report of a ten year old boy presenting with microscopic hematuria of glomerular origin and a medical history of orbital pseudotumor two years before his hospitalization due to renal symptoms. Renal biopsy revealed lesions of pauci-immune glomerulonephritis and findings of granulomatous inflammation and necrotizing vasculitis. Serum was positive for p-ANCA antibodies (perinuclear staining pattern ANCA antibodies). These findings led to the diagnosis of WG of generalized form (according to EULAR/ PRINTO/PRES criteria). The patient has been treated with aggressive immunotherapy with the use of steroids, cyclophosphamide and mycophenolate mofetil. Disease remission has been established and retained one year after initial diagnosis. Orbital pseudotumor, which is a diagnosis of exclusion, has been the initial disease's clinical manifestation, even though at that time neither the ocular biopsy nor the immunologic workup had been indicative in terms of WG. Although WG is very rare in children, this disease should always been included in the differential diagnosis in patients with similar clinical manifestations and clinicians should emphasize on the recognition of *Corresponding author.
\end{abstract}

granulomatous vasculitis in biopsies as well as on repeated tests for ANCA antibodies' detection in serum. High morbidity and mortality rates [1] of this clinical entity necessitates the early recognition of atypical disease's forms and the close follow up in cases of uncertain initial diagnosis.

Keywords: Pseudotumor; Hematuria; Wegener Granulomatosis; ANCA; Childhood

\section{INTRODUCTION}

Wegener granulomatosis (WG) is a necrotizing granulomatous vasculitis that affects small to medium sized vessels and is characterized by the presence of anti-neutrophil cytoplasmic antibodies (ANCA associated vasculitis) [1].

WG is rare in children. The incidence rate of WG ranges between 0.03 and 3.2 per 100,000 children per year [2]. During childhood, the majority of WG cases are Caucasian female adolescents. The most commonly affected vital organs are the kidneys [3]. Without therapy, the overall mortality of WG at 1 year has been estimated approximately to $80 \%$ [3]. Elderly patients have considerably higher early mortality [3]. With the therapeutic use of cyclophosphamide, however, there have been reported 1- and 5-year survival rates of $85 \%$ and $75 \%$ respectively [3]. Age and high serum creatinine levels at the time of initial diagnosis are the stronger worst predictors for both patient and renal survival [4]. Moreover, patients with severe disease upon initial diagnosis and vasculitis relapses during the follow up period are more likely to have a poor outcome [4]. Regarding the morbidity and mortality of WG in children, major issues are the hazards from a possible diagnostic delay, the toxicity of treatment and the propensity of ANCA associated vasculitis to relapse [3]. 
We describe a case of a ten year old boy presenting with microscopic hematuria as the major clinical manifestation before the diagnosis of Wegener granulomatosis. However, hematuria has not been the first clinical manifestation of WG in this patient, as two years earlier this child had been hospitalized for the diagnosis and management of orbital pseudotumor. Ocular manifestations of WG may include scleritis, conjunctivitis, uveitis, optic neuritis and retro-orbital pseudotumor [1]. In pediatric studies of WG, ocular involvement has been reported in percentages between $10 \%$ - 50\% [5-7].

Taking into account the significant morbidity and mortality of WG, we aim to highlight the importance of atypical disease's forms early recognition and treatment as well as the close follow-up of patients in which the initial diagnosis is uncertain.

\section{CASE REPORT}

A ten year old boy has been referred to a central general children's hospital for the diagnostic investigation of microscopic hematuria of glomerular origin accompanied by impaired renal function (serum urea: $79 \mathrm{mg} / \mathrm{dl}$, [normal values: 5 - $18 \mathrm{mg} / \mathrm{dl}$ ] and serum creatinine: $1.7 \mathrm{mg} / \mathrm{dl}$, [normal values: $0.5-1.0 \mathrm{mg} / \mathrm{dl}])$ as well as anemia ( $\mathrm{Hb}$ : $8.9 \mathrm{~g} / \mathrm{dl}$, [normal values: 10.3 - $14.9 \mathrm{~g} / \mathrm{dl}$ ]). Fifteen days earlier the child had fever for five days, pharynx inflammation and scarlet fever like rash on his trunk. A clinical diagnosis of a streptococcal upper respiratory tract infection has been made and the patient has been prescribed to cefprozil. On the third day of the fever, upon laboratory workup it has been detected the presence of microscopic hematuria. The assiduous clinical examination has only revealed a mild skin paleness. The height and weight were on the $50^{\text {th }}-75^{\text {th }}$ percentile and blood pressure was normal.

From the patient's medical history it has been noted that he had been hospitalized two years earlier, due to blepharoptosis. The differential diagnosis included orbital lymphoma, metastatic carcinoma, sarcoidosis, Langerhans cells histiocytosis (LCH), carotid-cavernous fistula (CCF), Graves' disease, orbital bacterial cellulitis, tuberculosis, myasthenia and Wegener's granulomatosis [8]. The laboratory tests in terms of malignancy and infections as well as the immunologic workup (RA-test, ANA, anti-ds DNA, p-ANCA, c-ANCA, CH-100, C3, C4) were negative. The orbital CT and MRI scanning had demonstrated a discrete orbital lesion with well defined borders, without any muscle or bone structures' infiltration. An ocular biopsy had been performed and the histological findings had not been indicative of any of the aforementioned pathologies that had been included in the initial differential diagnosis including WG. The diagnosis of retro-orbital pseudotumor had been made and the boy had been treated with pseudotumor partial surgical removal and high corticosteroids administration.

From the family medical record, it has been noted that the patient's father was suffering from psoriatic arthritis and his mother from Sjogren disease.

The initial laboratory workup revealed microcytic anemia (Hb: $8 \mathrm{~g} / \mathrm{dl}$, [normal values: 10.3 - $14.9 \mathrm{~g} / \mathrm{dl}$ ]), increased erythrocyte sedimentation rate (ESR: $70 \mathrm{~mm}$ /hour, [normal values: 4 - $20 \mathrm{~mm} /$ hour]), increased levels of urea $(76 \mathrm{mg} / \mathrm{dl}$, [normal values: $5-18 \mathrm{mg} / \mathrm{dl}])$ and creatinine $(1.6 \mathrm{mg} / \mathrm{dl}$, [normal values: $0.5-1.0 \mathrm{mg} / \mathrm{dl}])$ in serum, as well as a mild reduction of the serum protein and albumin levels. Urine microscopy with the use of phase contrast technique revealed erythrocytes of glomerular origin. In addition, proteinuria (1.2 - $1.5 \mathrm{~g} / 24$ hours, [normal value for a child: $<150 \mathrm{mg} / 24$ hours]) was found in several measurements. Both C3 and ASTO titres were high, ruling out the diagnosis of acute post-streptococcal glomerulonephritis, whereas C4, ANA, anti-DNA, Ra-test and thyroid tests were normal. Moreover, there has been found hypergammaglobulinemia and high IgG serum titres. Blood cultures for common bacteria and serological tests for other infections caused by viruses (HIV, HBV, HCV) or mycoplasma were negative. The immunological tests were positive for the p-ANCA-anti-MPO antibodies but negative for the c-ANCA-anti-PR3 antibodies. Cardiac ultrasound, chest X-ray and ocular examination were normal. Renal ultrasound revealed bilateral increased echogenicity of renal cortex, compatible with the presence of mild chronic kidney disease.

The differential diagnosis included the causes of microscopic hematuria in terms of a systemic disease with normal levels of C3, i.e. Goodpasture syndrome, HenochSchonlein purpura, hemolytic uremic syndrome, polyarteritis nodosa and ANCA-associated vasculitis [9]. Renal biopsy revealed the presence of focal glomerular necrosis and extracapillary proliferation in the absence of significant glomerular immune deposits, i.e. an histological picture of pauci immune glomerulonephritis. Moreover, there were findings of granulomatous inflammation and necrotizing vasculitis. According to the EULAR/PRINTO/ PRES criteria [10], the diagnosis was Wegener granulomatosis of generalized type [11].

Disease categorization $[11,12]$ determines the options in patients' therapeutic management. The remission induction therapy included six pulses of methyl-prednisolone in every second day with i.v. administration (1000 $\mathrm{mg} / 1.73 \mathrm{~m}^{2}$ ) followed by tapering of corticosteroids with p.o. prednisolone administration ( $1 \mathrm{mg} / \mathrm{kg} / 24 \mathrm{hrs})$ as well as six pulses of cyclophosphamide (500 $\mathrm{mg} / \mathrm{m}^{2} /$ month). Disease remission, according to WG validated criteria [10], has been attained after six months. The second stage treatment included corticosteroids tapering and the use of mycophenolate mofetil (540 mg per day) in terms 
of remission maintenance therapy.

The patient's treatment procedure has been successful as remission has been attained [10] and moreover has been maintained one year later. During last patient's follow up, the urine tests were normal, the protein to creatinine ratio in a first morning voided specimen was 0.25 [normal value for children above 2 years olds: $<0.2$ ], serum urea was $59 \mathrm{mg} / \mathrm{dl}$ [normal values: 5 - $18 \mathrm{mg} / \mathrm{dl}$ ], serum creatinine $1 \mathrm{mg} / \mathrm{dl}$ [normal values: 0.5 - $1.0 \mathrm{mg} / \mathrm{dl}$ ], ESR was $5 \mathrm{~mm} /$ hour [normal values: 4 - $20 \mathrm{~mm} /$ hour] and $\mathrm{Hb}$ was $12.5 \mathrm{~g} / \mathrm{dl}$ [normal values: 10.3 - $14.9 \mathrm{~g} / \mathrm{dl}$ ].

\section{DISCUSSION}

We describe the case of a ten year old child presenting with microscopic hematuria of glomerular origin and impaired renal function. Anemia and increased ESR as well as the presence of orbital pseudotumor from the patient's medical history led the differential diagnosis towards a multi systemic disease. Multi systemic diseases with low C3 serum levels that present with microscopic hematuria [9] include the following clinical entities: hemolytic uremic syndrome, Goodpasture syndrome, Henoch-Schonlein disease, polyarteritis nodosa as well as ANCA associated vasculitis. The former category of vasculitis includes Churg Strauss disease which is characterized by the presence of asthma and eosinophilia, Wegener granulomatosis (WG) and microscopic polyangiitis (MPA) [13]. The detection of pauci immune necrotizing glomerulonephritis in renal biopsy characterizes WG as well as MPA whereas the presence of granuloma in affected organ's biopsy - in this case in renal biopsy-is only found in WG. Moreover, orbital pseudotumor itself is a typical finding only in the case of WG. However, the distinction between those two clinical entities is often difficult. In WG there are most commonly detected c-ANCAs which present with a diffusely granular, cytoplasmic staining pattern-directed against PR3. However, there can also been detected p-ANCAs, that are most commonly found in MPA, and which present with a perinuclear staining pattern-directed against MPO [14]. The accurate diagnosis was based on the presence of pauci-immune necrotizing glomerulonephritis in combination with the granulomatous inflammation in the renal biopsy.

The most commonly affected organs [1], in adults as well as in children, are upper and lower respiratory tract, kidneys, eyes, central nervous system and skin. Clinical manifestations from the respiratory tract include saddle-nose deformity due to a perforated septum, rhinosinusitis, glottitis and airway stenosis, polyps, lung granulomatous nodes, lung hemorrhage. Renal involvement includes pauci-immune necrotizing glomerulonephritis and the following findings: proteinuria, hematuria, renal insufficiency and hypertension. The most common ocular manifestations are ocular granulomas, retina hemorrhage, pseudotumors, scleritis, conjunctivitis, uveitis, episcleritis. Among central nervous system disorders, the most common are peripheral nervous or central nervous palsy, brain hemorrhage, non infectious meningitis. Skin rashes include purpura and nodules. Less common clinical manifestations derive from the joints, the cardiovascular system, the gastrointestinal tract, the gonads, the breast and the salivary glands. There can also be noted general symptoms such as malaise, fever, weight loss [1].

Orbital pseudotumor (idiopathic orbital inflammation) is an idiopathic, nonspecific inflammation of any of the orbital structures [8]. It should be always considered as a diagnosis of exclusion, with evaluation directed toward eliminating other causes of orbital disease or underlying systemic disease. It is usually unilateral, males and females are equally affected and there is no racial predilection. Onset is most frequently in middle age but can occur at any age. Some children and young adults who develop orbital pseudotumor have had a preceding viral infection. Moreover, it is possible that there may be a relation with an underlying immune reaction as the majority of children with orbital pseudotumor eventually develop autoimmune systemic diseases, such as WG [15].

WG classification according to the European Vasculitis Study (EUVAS) is very important for the disease treatment and prognosis [11]. WG may present by the following forms:

1) Localized, with upper and/or lower respiratory tract disease without any other systemic involvement or constitutional symptoms,

2) Early systemic, in which any organ may be affected but without organ threatening disease or life threatening disease,

3) Generalized, with renal or other organ threatening disease, serum creatinine below $5.6 \mathrm{mg} / \mathrm{dl}$,

4) Severe, with renal or other vital organ failure and serum creatinine above $5.6 \mathrm{mg} / \mathrm{dl}$,

5) Refractory, which refers to progressive disease unresponsive to glucocorticoids and cyclophosphamide.

WG management includes the induction therapy and the maintenance therapy whereas the therapeutic agents in each stage depend on the disease classification [12]. In our patient's case who suffered from a generalized form of WG, the first stage induction therapy included glucocorticoids and cyclophosphamide whereas the second stage maintenance therapy incuded low glucocorticoids dosage together with mycophenolate mofetil. The aim of maintenance therapy is the perevention of relapses. It is of high significance the prophylactic use of therapeutic means against possible toxic adverse reactions of the immunosuppressive medication in WG. The prophylactic 
use of antibiotics against opportunistic infections (Pneumocystis carinii), vaccination against flu and pneumococcus before treatment as well as the use of prophylactic means against specific adverse effects of medication (e.g. 2-mercaptoethane sulfonate for preventing hemorrhagic cystitis if cyclophosphamide is used) should always be a high priority in WG management [3].

The prognosis of children with WG depends not only from the disease severity but also from the medication's adverse reactions [1]. Disease related morbidity mainly derives from subglottic stenosis and renal insufficiency. From studies performed in children with WG, it has been demonstrated that relapse rates after remission maintenance fluctuate between $53 \%$ - $80 \%$ whereas in the same studies mortality rate is estimated to $2 \%$ [1]. Drug related morbidity includes short term adverse reactions such as nausea and vomiting, cytopenia, elevation of liver function tests, renal dysfunction, opportunistic infections, hemorrhagic cystitis as well as long term complications such as osteoporosis, folic acid insufficiency, infertility and cancer [16]. In children the most common complications derived from toxic therapies include infertility (28\% - 40\%), cyclophosphamide related cystitis (50\%) and serious opportunistic infections (43\%), mainly pneumonia from Pneumocystis carinii, whereas malignancies are not usually found in children [1].

Early diagnosis and management of children with WG as well as close follow up and careful monitoring of therapy's complications are crucial for patients' life expectancy as well as for their quality of life.

\section{REFERENCES}

[1] Frosch, M. and Foell D. (2004) Wegener granulomatosis in childhood and adolescence. European Journal of Pediatrics, 163, 425-434. doi:10.1007/s00431-004-1464-3

[2] Cabral, D.A., et al. (2009) Classification, presentation, and initial treatment of Wegener's granulomatosis in childhood. Arthritis \& Rheumatism, 60, 3413-3424. doi:10.1002/art.24876

[3] Jayne, D. (2009) Review article: Progress of treatment in ANCA-associated vasculitis. Nephrology (Carlton), 14, 42-48. doi:10.1111/j.1440-1797.2009.01101.x
[4] Westman, K.W., et al. (1998) Relapse rate, renal survival, and cancer morbidity in patients with Wegener's granulomatosis or microscopic polyangiitis with renal involvement. Journal of the American Society of Nephrology, $\mathbf{9}$ 842-852.

[5] Roberti, I., Reisman, L. and Churg, J. (1993) Vasculitis in childhood. Pediatric Nephrology, 7, 479-489. doi:10.1007/BF00857580

[6] Rottem, M., et al. (1993) Wegener granulomatosis in children and adolescents: Clinical presentation and outcome. Journal of Pediatrics, 122, 26-31. doi:10.1016/S0022-3476(05)83482-1

[7] Stegmayr, B.G., et al. (2000) Wegener granulomatosis in children and young adults. A case study of ten patients. Pediatric Nephrology, 14, 208-213. doi:10.1007/s004670050043

[8] Atkin, S.R. (1990) Orbital pseudotumor, differential diagnosis. Optometry \& Vision Science, 67, 840-844. doi:10.1097/00006324-199011000-00009

[9] The Johns Hopkins Hospital (2008) The Harriet Lane handbook: A manual for Pediatric House Officers.

[10] Vanoni, F., et al. (2010) Vasculitides associated with IgG antineutrophil cytoplasmic autoantibodies in childhood. Pediatric Nephrology, 25, 205-212. doi:10.1007/s00467-009-1253-3

[11] Mukhtyar, C., et al. (2009) EULAR recommendations for the management of primary small and medium vessel vasculitis. Annals of the Rheumatic Diseases, 68, 310317. doi:10.1136/ard.2008.088096

[12] Pallan, L., Savage, C.O. and Harper, L. (2009) ANCAassociated vasculitis: From bench research to novel treatments. Nature Reviews Nephrology, 5, 278-286. doi:10.1038/nrneph.2009.45

[13] Rigante, D. (2006) Clinical overview of vasculitic syndromes in the pediatric age. European Review for Medical and Pharmacological Sciences, 10, 337-345.

[14] Nash, M.C. and Dillon, M.J. (1997) Antineutrophil cytoplasm antibodies and vasculitis. Archives of Disease in Childhood, 77, 261-264. doi:10.1136/adc.77.3.261

[15] Brown D.H, MacRae, D.L. and Allen, L.H. (1998) Orbital Pseudotumors. Journal of Otolaryngology, 17, 164168.

[16] Wung, P.K. and Stone, J.H. (2006) Therapeutics of Wegener's granulomatosis. Nature Clinical Practice Rheumatology, 2, 192-200. doi:10.1038/ncprheum0139 